\title{
Measuring nanoscale friction at graphene step edges
}

\author{
Zhe CHEN, Seong H. KIM* \\ Department of Chemical Engineering and Materials Research Institute, Pennsylvania State University, University Park, PA 16802, USA \\ Received: 02 May 2019 / Revised: 20 August 2019 / Accepted: 09 October 2019 \\ (C) The author(s) 2019.
}

\begin{abstract}
Although graphene is well known for super-lubricity on its basal plane, friction at its step edge is not well understood and contradictory friction behaviors have been reported. In this study, friction of mono-layer thick graphene step edges was studied using atomic force microscopy (AFM) with a Si tip in dry nitrogen atmosphere. It is found that, when the tip slides over a 'buried' graphene step edge, there is a resistive force during the step-up motion and an assistive force during the step-down motion due to the topographic height change. The magnitude of these two forces is small and the same in both step-up and step-down motions. As for the 'exposed' graphene step edge, friction increases in magnitude and exhibits more complicated behaviors. During the step-down motion of the tip over the exposed step edge, both resistive and assistive components can be detected in the lateral force signal of AFM if the scan resolution is sufficiently high. The resistive component is attributed to chemical interactions between the functional groups at the tip and step-edge surfaces, and the assistive component is due to the topographic effect, same as the case of buried step edge. If a blunt tip is used, the distinct effects of these two components become more prominent. In the step-up scan direction, the blunt tip appears to have two separate topographic effects-elastic deformation of the contact region at the bottom of the tip due to the substrate height change at the step edge and tilting of the tip while the vertical position of the cantilever (the end of the tip) ascends from the lower terrace to the upper terrace. The high-resolution measurement of friction behaviors at graphene step edges will further enrich understanding of interfacial friction behaviors on graphene-covered surfaces.
\end{abstract}

Keywords: friction; graphene; step edge; atomic force microscopy; tip bluntness

\section{Introduction}

Graphene, due to its superior strength and low friction, is an ideal material for ultra-thin protective coatings from nanoscale to macroscale [1-8]. In order to understand the friction mechanism of graphene, friction tests have extensively been performed using atomic force microscopy (AFM) for a single point contact between the ultra-sharp AFM tip and the graphene. It was found that friction on the flat graphene basal plane mainly depends on the graphene thickness [9-16] and the interaction between the graphene and the substrate [13-16]. These parameters influence the puckering of the graphene basal plane and further influence the friction. However, flat graphene basal planes cannot represent entire graphene coatings at macroscales; coatings over a large area will contain defects such as wrinkles, folds, step edges, humps, and so on. Among the structures mentioned above, the graphene step edge plays significant roles in friction because of its relatively high abundance and extremely large friction compared to the basal plane [17-30].

When the AFM tip ascends a step edge, the friction always increases to a level more than an order of magnitude larger than that of the basal plane [17-30]. In the descending case, a majority of previous works showed that there is a resistive force acting against the tip sliding, increasing friction [17-21, 23, 26-30],

* Corresponding author: Seong H. KIM, E-mail: shkim@engr.psu.edu 
while some other researchers reported that the friction force decreases and even becomes negative [21, 24]. The negative friction means that, due to the topography, the AFM tip is pushed forward in the descending direction by the step edge. Those who observed the decrease in friction as the tip descends at the step edge believe that the geometric effect due to the topographic height change is the dominant factor for friction at the step edge [24]. In contrast, those who reported the increase in friction hold the opinion that the topography effect is negligible and propose the existence of a large additional potential barrier at the step edge [21, 27]. This concept was borrowed from the Schwoebel-Ehrlich barrier which was originally introduced to explain the surface diffusion of atoms on stepped surfaces [31]. In the Schwoebel barrier model for friction, the potential at the higher terrace near the step edge is larger than the value away from the step edge, but the potential at the lower terrace near the step edge is smaller [31]. Therefore, during the step-down process, a larger traction force is needed to pull the tip over the potential barrier at the higher terrace and out of the potential well at the lower terrace, thus resulting in higher friction [21, 27]. Another mechanism attributes the increased friction to the dangling bonds and other functional groups at the graphene step edge, which may have attractive interactions with the AFM tip and generate resistance to sliding motion [19, 22, 29]. Some molecular dynamics (MD) simulations and density functional theory (DFT) calculations supported the first mechanism that the extra potential barrier is dominating the friction at the graphene step edge [21, $27,32]$, but chemical reactions were not considered in those computations. Considering that the friction at the graphene step edge can be orders of magnitude higher than that on the graphene basal plane, it is important to better understand the frictional behavior of the graphene step edge.

In this work, we report how to precisely measure friction at the graphene step edge with AFM. Both exposed and buried single-layer graphene step edges were found on a freshly-exfoliated graphite surface. Then, the effects of AFM scanning resolution and tip bluntness on the obtained lateral signal at exposed single-layer graphene step edges were analyzed. The data presented here provide an insight into the fundamental processes governing friction at the graphene step edge and explain why contradictory results were reported before. The method presented in this paper would also be useful for interfacial friction measurements on topographically-corrugated and chemically-complex surfaces.

\section{Materials and methods}

A freshly-cleaved graphite surface was produced on a highly-oriented pyrolytic graphite (HOPG) crystal by tape-exfoliation in ambient air. Nanoscale friction tests were performed using an AFM system (Multimode, Bruker, US) with Si tips (CONTV, Bruker, US) in the contact scan mode. Before the test, the AFM tip was treated with UV/ozone for $15 \mathrm{~min}$ [33]. During the test, the reciprocating frequency in the lateral direction was fixed at $2 \mathrm{~Hz}$, the scan size varied from $100 \mathrm{~nm}$ to $4 \mu \mathrm{m}$, so the tip sliding speed ranged from $200 \mathrm{~nm} / \mathrm{s}$ to $8 \mu \mathrm{m} / \mathrm{s}$. The applied normal force on the AFM tip was in the range from 5 to $20 \mathrm{nN}$. The normal spring constant of the AFM probe cantilever was calibrated following the Sader's method [34]. The lateral sensitivity of the cantilever and detector was calculated by comparing the measured lateral signal (in the unit of $\mathrm{mV}$ ) on a reference sample with the known coefficient of friction (COF). The reference sample was a diamond-like carbon (DLC) coating which possesses a COF of $\sim 0.15$ in a pentanol vapor lubrication condition, where the partial pressure is $40 \%$ of the saturation pressure [35]. All tests were conducted at room temperature (22$25^{\circ} \mathrm{C}$ ). Dry nitrogen was passing through the AFM chamber at the rate of $30 \mathrm{~cm}^{3} / \mathrm{min}$ during the friction test. Both topography and lateral signals were collected at the same time. The blunt AFM Si tip was obtained by sliding it against a Si wafer in ambient air [36]. A scanning electron microscope (SEM, Scios 2, Thermo Fisher Scientific, US) was used to take images of the AFM tips, with a primary beam energy of $5 \mathrm{kV}$, a beam current of $0.4 \mathrm{nA}$, and a working distance of about $10 \mathrm{~mm}$.

\section{Results and discussion}

Figure 1(a) shows the topography and corresponding lateral signal on trace and retrace scans of a square 
region on the freshly-exfoliated graphite surface. Based on the topography, one can identify many features on the graphite surface, including step edges, wrinkles, and humps. Some of these features, such as the wrinkle marked in Fig. 1(a), are hard to notice in the topography image, but they exhibit lateral signals quite distinct from the basal plane. Some other features, such as the hump marked in Fig. 1(a), can be easily found in the topography image, but have little contribution to the lateral signal; this is because the inclination angle of the hump slope is only $0.25^{\circ}$ and the height changes very gradually. Among all the features, step edges are the most common and obvious ones in the topography image. However, some of them (marked with solid-line arrows in Fig. 1(a)) have a strong lateral force signal, while others (marked with dot-line arrows in Fig. 1(a)) have a very weak lateral signal.

In order to further investigate the lateral signal of graphene step edges, the dash-box region in Fig. 1(a) was scanned with a higher resolution and the data are displayed in Fig. 1(b). It can be seen that there are three step edges in this region, and they are schematically re-drawn in Fig. 1(c) and marked as A, $\mathrm{B}$, and $\mathrm{C}$. The height profiles along the solid lines in Fig. 1(b) are plotted in Fig. 1(d). All three step edges are associated with a height change $(\sim 0.3 \mathrm{~nm})$ corresponding to the thickness of one single-layer graphene.

The lateral signals along the dash-lines, which are marked as Line 1 and Line 2 in Fig. 1(b), are plotted in Figs. 1(e) and 1(f), respectively. During the trace scanning, in which the AFM tip slides from left to right, the AFM tip ascends step-A and descends step-B (a)

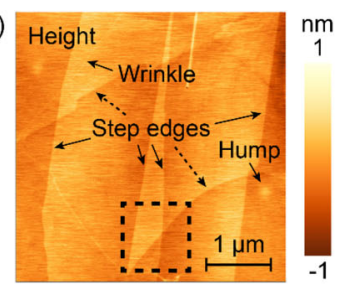

(b)

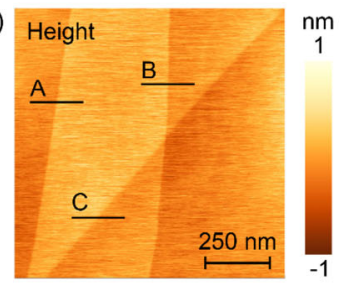

(c)

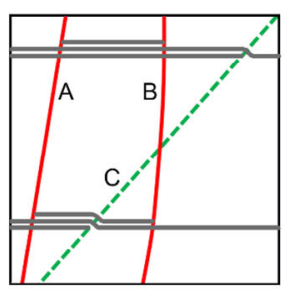

(d)

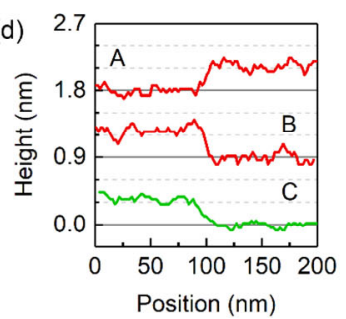

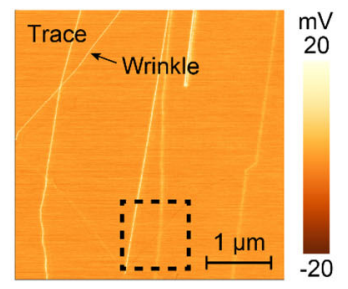
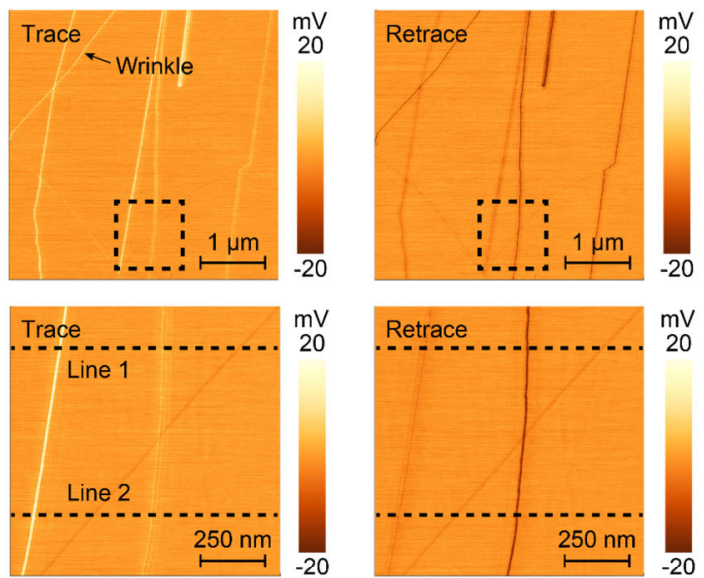

(e)

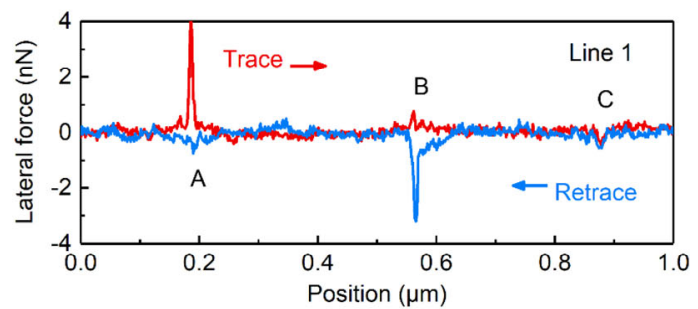

(f)

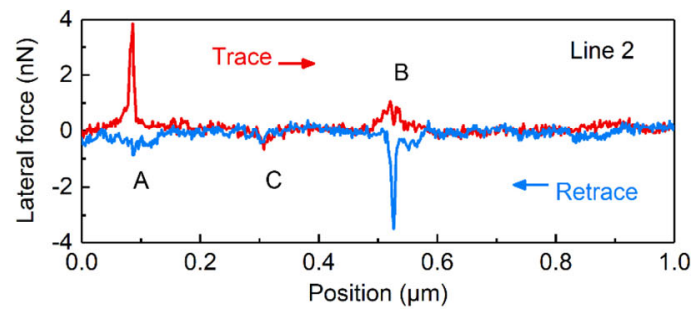

Fig. 1 Topography and lateral signal collected by AFM with contact mode on a graphite surface. (a) Height and lateral signal (trace and retrace) images of a $4 \mu \mathrm{m} \times 4 \mu \mathrm{m}$ area. (b) Height and lateral signal (trace and retrace) images of a $1 \mu \mathrm{m} \times 1 \mu \mathrm{m}$ area marked with a dashed box in (a). (c) Illustration showing step edges identified in (b), with the red lines indicating exposed step edges and the green line indicating the buried step edge. (d) Height line profiles along the solid-lines across the three steps marked as A, B, and C in (b). (e, f) Lateral signal line profiles marked with dash-lines (Line 1 and Line 2) in (b). 
and step-C. The opposite takes place during the retrace scanning. Note that the sign of the lateral signal changes as the AFM tip changes the scan direction; in other words, the positive lateral signal during the trace scanning and the negative lateral signal during the retrace scanning indicate that the tip encounters resistive force against sliding. It can be found that, on the graphene basal plane, the trace and retrace lateral signals almost overlap with each other. This implies that the friction between the AFM tip and the graphene basal plane is very small, which agrees with previous results [37].

In Figs. 1(e) and 1(f), it can be seen that the lateral signals at step-A and step-B are similar to each other except the opposite signs due to the difference in the height change direction; however, the lateral signals at step-C are quite different from those at step-A and step-B despite the fact that they all have the same topographic height change $(\sim 0.3 \mathrm{~nm})$. For step-A and step-B, no matter the AFM tip ascends or descends the graphene step edge, the magnitude of the trace and retrace lateral signals increase to the opposite directions away from the zero baseline, which indicates that there is always larger traction at these steps compared to the basal planes. As for step-C, the lateral signals during trace and retrace scans shift to the same direction, and the magnitude is barely larger than the noise level of the basal plane lateral signal. The shift of the lateral signal in the same direction in the trace and retrace is typical of the topographic effect [38, 39]. When the tip ascends a slope, there is a resistive force to tip sliding; when the tip descends the same slope in the retrace, the tip experiences an assistive force facilitating the sliding along the slope. Since the scan direction is opposite, the resistive force in the trace and the assistive force in the retrace shift the measured lateral signal in the same direction (as in the case of step-C in Figs. 1(e) and $1(\mathrm{f}))$. Such phenomena were well documented in Refs. [19, 21-23]. When a single-layer thick step edge is covered with another graphene layer, the tip sliding over that overlayer would be very insensitive to any chemical functional groups present at the step edge. Thus, it is reasonable to assign step-C to a 'buried' step (or a step covered with another graphene layer; as schematically illustrated in Fig. 1(c)).

The difference in the lateral signal pattern between steps-A and B versus step-C indicates that steps-A and
B cannot be buried ones, they must be 'exposed' steps (schematically illustrated in Fig. 1(c)). It is clearly noted that the friction during descending (trace case of step-B and retrace case of step-A in Figs. 1(e) and 1(f)) is resistive although its magnitude is not as big as the friction during ascending (trace case of step-A and retrace case of step-B in Figs. 1(e) and 1(f)). The origin of this resistive force during descending remains controversial $[19,21,22,27]$. Moreover, the shape of the friction signal during descending reported in the literature also varied drastically - in some papers, the friction profile was a single peak $[19,20,22,23,26,27$, $30]$, but in some others, the friction profile seemed to be composed of two peaks with a valley between them $[20,21,28,29]$. For better understanding the origin of the resistance during descending, it is critical to resolve this discrepancy.

To further investigate the exact shape of the friction signal at the exposed graphene step edge, a region with several exposed step edges almost perpendicular to the AFM tip fast-scan direction was selected and scanned at different data collection resolutions (nm per pixel). Figure 2(a) shows the topography, and trace and retrace lateral signals collected with an applied normal force of $5 \mathrm{nN}$. The scan size was $4 \mu \mathrm{m}$ in the fast-scan direction and $1 \mu \mathrm{m}$ in the slow-scan direction. Considering that each fast-scan line consists of 512 pixels, the scan resolution was about $7.81 \mathrm{~nm}$ per pixel. Based on the height change in the topography image, two step edges in the right portion of the image are found to be single-layer graphene step edges. The height increases from left to right, so the AFM tip ascends these two step edges during the trace scanning and descends during the retrace scanning. The lateral signals along the red and blue lines in Fig. 2(a) are plotted in Fig. 2(e). At each graphene step edge, there is a large friction increase in both trace (positive lateral signal) and retrace (negative lateral signal) directions. It is very similar to the results reported by some previous Refs. [19, 20, 22, 23, 26, 27, 30].

Then, the region containing the same two exposed single-layer graphene step edges, which is marked with a dash-box in Fig. 2(a), was scanned with a higher resolution. The scan size was reduced to $1 \mu \mathrm{m}$ in the fast-scan direction and $250 \mathrm{~nm}$ in the slow-scan direction, while the total number of pixels collected in each fast-scan line was kept constant at 512. Thus, the scan 
resolution was increased to $1.95 \mathrm{~nm}$ per pixel. The topography and lateral signal images are displayed in Fig. 2(b), and the lateral signals along the dash-lines at the same single-layer graphene step edges are plotted in Fig. 2(f). Comparison of the lateral signals in Figs. 2(e) and 2(f) shows that, except the increased noise, while the trace lateral signal shows no significant change, the retrace lateral signal at the high resolution (Fig. 2(f)) reveals an extra weak peak pointing to the positive direction, which is not observed in the low-resolution image (Fig. 2(e)).

To check whether the very weak extra peak is signal or noise, the region marked with dash-box in Fig. 2(b) was scanned one more time. This time the scan size was $200 \mathrm{~nm}$ in the fast-scan direction and $50 \mathrm{~nm}$ in the slow-scan direction, and the scan resolution increased to $0.39 \mathrm{~nm}$ per pixel. The obtained topography and lateral signal images are shown in Fig. 2(c) and the lateral signal along the dash-lines are plotted in Fig. 2(g). It can be found that, with higher scan resolution, the peak at the bottom of the retrace lateral signal region becomes even more prominent. Thus, the positive peak, which can only be observed under relatively high scan resolution, can be confirmed to be real signal. This finding implies that the previous Refs. [19, 20, 22, 23, $26,27,30]$, in which the scan resolution was not high enough, might have missed the fine features in the friction response of the single-layer graphene step edge during the step-down scan.

In order to eliminate the scan noise, which is often substantial when a soft cantilever with a low spring constant is used, the slow scan of the AFM imaging was turned off and a set of 128 line scans along the black dash-line in Fig. 2(c) was recorded with a scan resolution of about $0.20 \mathrm{~nm}$ per pixel $(100 \mathrm{~nm} / 512$ pixels). The scanning data are presented in Fig. 2(d), and the average of the 128 line-scan data is shown in Fig. 2(h). After the noise is reduced by averaging the line-scan data, it is clear that the lateral signal data in both ascending (red colored) and descending (blue colored) directions have two components: a broad component with opposite signs in opposite scan directions (marked with dash-lines) and a sharp component with the same sign in both trace and retrace directions (marked with dot-lines).

In the trace lateral signal, these broad and sharp components are in the resistive force direction, implying that they act against the ascending motion of the AFM tip at the graphene step edge. In the retrace lateral signal, while the broad component is in the resistive force direction, the sharp component is assistive meaning that it facilitates the descending motion of the AFM (a)

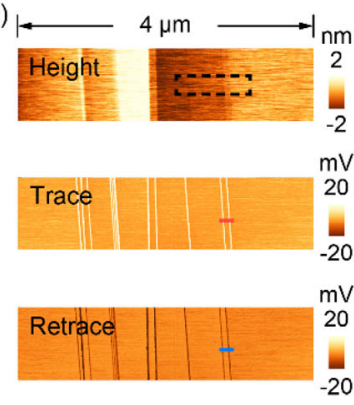

(e)

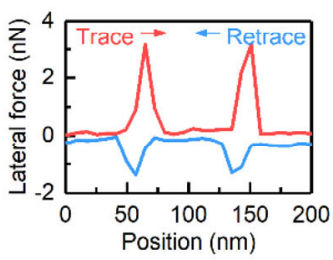

(b)

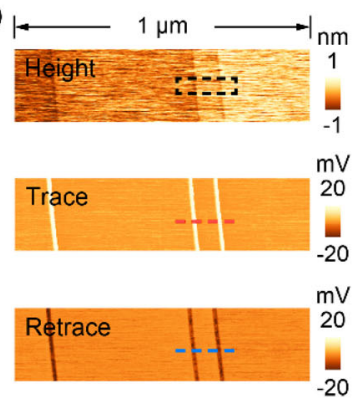

(f)

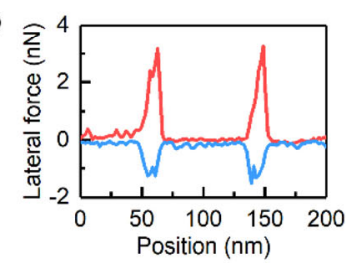

(c)

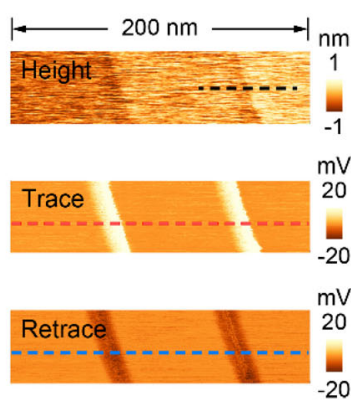

(g)

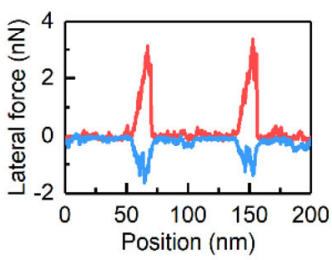

(d)

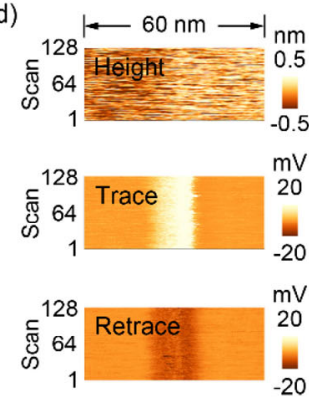

(h)

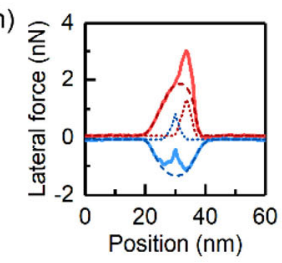

Fig. 2 Revealing fine features in the friction response of $0.3 \mathrm{~nm}$ thick single-layer graphene step edges exposed to air. (a) shows the topography, and trace and retrace lateral signal images of a $4 \mu \mathrm{m} \times 1 \mu \mathrm{m}$ area. (b) shows the data collected in the dash-box in (a), and (c) shows the data collected in the dash-box in (b). (d) shows the data of 128 scans along the black dash-line in (c). The scan rate was kept constant at $2 \mathrm{~Hz}$ and each scan line consists of 512 pixels. The trace and retrace lateral signals shown in (e-g) are from the single line profile marked in $(\mathrm{a}-\mathrm{c})$, respectively. $(\mathrm{h})$ shows the lateral signal line profile averaged from the 128 -scan data in (d). The lateral signal in (h) is deconvoluted into two components. The applied normal load between the tip and the graphite surface was $5 \mathrm{nN}$. 
tip from the upper terrace to the lower terrace. The fact that the sharp components in the trace and retrace scans have the same sign in the lateral signal of the AFM scan means that they originate from the topographic effect (similar to the buried step edge case shown in Figs. 1(e) and 1(f)) [17, 18]. The resistive force corresponding to the broad components must originate from differences in chemistry at the exposed step edge compared to the basal plane $[17,18]$, which is lacking in the buried step edge case because it is covered with the basal plane of graphene. Larger friction is typically expected in the region where stronger or favorable chemical interactions between two solid surfaces exist $[40,41]$. From the spectroscopic analysis and simulations, it has been shown that the exposed graphene step edge is terminated with $\mathrm{OH}$ groups [17, 18], which can have hydrogen bonding interactions with the hydrophilic silicon oxide counter surface [42]. Such hydrogen bonding interactions do not exist on the basal plane of graphene.

Whether the lateral force during the downward scan at the exposed graphene step edge will be entirely resistive or could be assistive depends on the relative magnitudes of the chemical (broad) and topographic (sharp) components. In Fig. 2(h), the magnitude of the assistive force due to the topography is smaller than that of the resistive force due to the chemical interactions, so the lateral force is entirely resistive. The data in Fig. 2(h) was collected at an applied load of $5 \mathrm{nN}$. When the applied load was increased to about $20 \mathrm{nN}$, as shown in Fig. 3(c), the assistive force due to the topography becomes larger than the resistive force originating from the chemical interactions. This means that the lateral force at the topographic transition region could be assistive, resulting in negative friction, which is similar to the descending case of the buried step edge. This also means that if the friction data is collected with a low resolution (similar to Fig. 1(a)) at a high load, it is possible that the resistive component could be missed, and the obtained data could be interpreted with the topography effect only [21, 24].

Since friction originates from the interaction between the AFM tip and the graphene step edge, the radius of the tip is a crucial factor to the friction signal. When the AFM tip is very sharp (Fig. 3(a)), the recorded friction signal in the ascending direction is so narrow that the topographical and chemical components are (a)
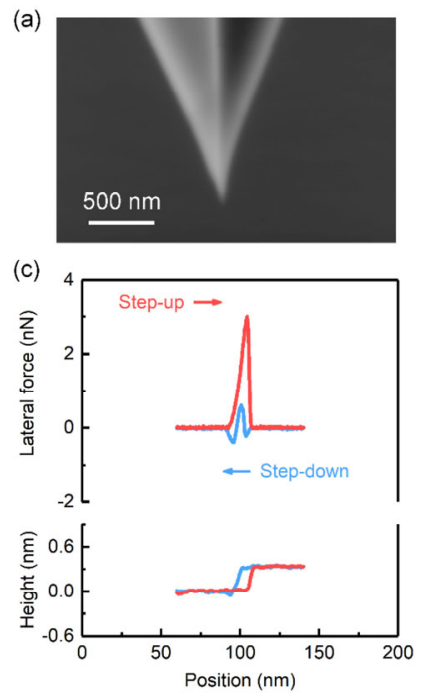

(b)
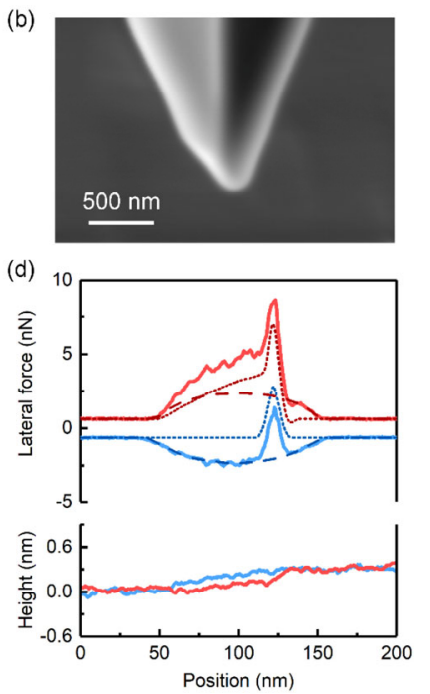

Fig. 3 Effect of tip bluntness on the lateral signal at the graphene step edge. Front view SEM images of (a) sharp and (b) blunt tips used for the experiment. Lateral signals and height profiles obtained with the (c) sharp and (d) blunt Si tips at an applied normal load of about $20 \mathrm{nN}$. The data collected during step-up motion is plotted in red color, and the data collected in step-down motion is plotted in blue color. The lateral signal shown in (d) are deconvoluted into two components, referring to chemical components (dash-lines) and topographical components (dot-lines), respectively.

both resistive and almost overlap with each other, making it difficult to deconvolute them (Fig. 3(c)). In the descending direction, the chemical effect is resistive and the topographic effect is assistive; thus, distinguishing these two components is relatively easy.

A blunt AFM tip could reveal much more easily the presence of two components in friction, although it may lose the topographic resolution. Figure 3(d) displays the lateral signal and height data collected with a blunt tip, whose image is shown in Fig. 3(b). At the same applied normal load, the magnitude of the friction on the basal plane and at the step edge is significantly larger for the blunt tip, compared to the sharp tip case (Fig. 3(c)). This phenomenon can be explained by the increased contact area leading to a larger adhesion force between the AFM tip and the sample surface $[43,44]$. Moreover, compared with the sharp tip, the distinction between the chemical and topographic components for the blunt tip is much more prominent, especially for the descending signal.

Considering that the diameter of contact area between the tip and the surface is much larger than the step edge width, and the topographic height change of the 
step edge is only $0.34 \mathrm{~nm}$ (for example, see Fig. 1 of Ref. [17]), the magnitude of the chemical components mainly depends on the length of the graphene step edge cross-sectioning the contact area between the AFM tip and the graphite surface. Therefore, for both ascending and descending processes, the resistive force due to chemical effects starts to increase as soon as the leading edge of the tip touches the graphene step edge, increases to a maximum value when the center of the contact area passes through the step edge, then gradually decreases as the center of the contact area moves away from the step edge, and becomes zero when the trailing edge of the tip leaves the graphene step edge. As for the topographical components, they are expected to be the largest or peaked when the center axis of the AFM tip crosses the step edge and thus the vertical position of the cantilever (which is recorded as the topography) changes. Therefore, the location of the sharp peak in the ascending friction signal would coincide with the height change in the topographic line profile.

For the lateral signal during the descending processes of the blunt tip, similar to the case shown in Fig. 2(h), the broad negative component is due to the chemical interaction (marked with blue dash-line in Fig. 3(d)), and the narrow positive component is the topographic contribution (marked with blue dot-lines in Fig. 3(d)). The magnitude of the chemical components during the ascending and descending processes must be similar because the same functional groups are involved. In fact, the number of hydrogen bonds formed between the tip surface and the step edge is found to be similar in both ascending and descending processes through reactive molecular dynamics (MD) simulations [17]. In Fig. 3(d), if the broad part of the ascending lateral signal is compared with the chemical component in the descending lateral signal, one can see that their magnitudes are different-the ascending case is significantly larger, which indicates that, besides the chemical effect, there is some other effect contributing to the broad component of the ascending lateral signal. Note that because the tip-surface contact diameter is much larger than the width of a graphene step edge over which the topographic height changes by $0.34 \mathrm{~nm}$, the force balance argument used in the wedge calibration method, in which the contact area can be regarded as a point on a slanted plane [39], may not be fully applicable here.

Alternatively, it can be conceived that before the center axis of the AFM tip crosses the graphene step edge, the leading edge of the AFM tip would undergo elastic deformation; this is schematically illustrated in Fig. 4. Before the center axis of the tip crosses the step edge, the recorded topographic height change is negligible; in other words, the tip is still moving horizontally (parallel to the basal plane). Similar to the chemical effect, the resistive force due to elastic deformation will be proportional to the length of the step edge cross-sectioning the contact area. Reactive MD simulations showed that the degree of atomic strains is larger during the ascending process than the descending process [17]. This might be the reason that the magnitude of the broad component is larger during the ascending than the descending in Fig. 3(d). Based on this argument, the lateral signal during the ascending process of the blunt tip can be deconvoluted into three components: (i) chemical interactions between the functional groups at the tip surface and the step edge, (ii) elastic deformation of the tip, and (iii) the geometry effect when the tip is physically lifted against the applied load. The third component is the same as the geometry effect in the wedge calibration of the lateral force [39]. The first component is marked with
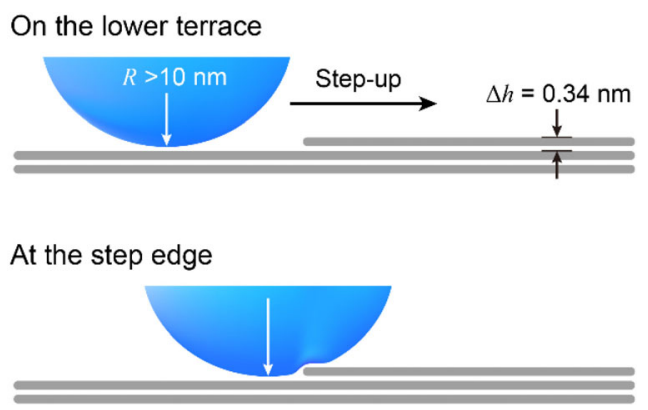

On the upper terrace

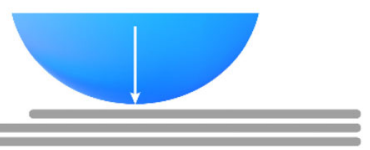

Fig. 4 Illustrations of an AFM tip slides across an exposed graphene step edge. The leading edge of the AFM tip elastically deforms when the AFM tip is at the graphene step edge. Note that the image is not in scale; the actual height of the step edge $(\Delta h)$ is much smaller than the radius $(R)$ of the tip. The white arrow is the center axis of the AFM tip. 
red dash-line in Fig. 3(d); the combined effect of the second and third components are marked with red dot-line in Fig. 3(d). This differentiation is possible because the topographic height change occurs only at a single line and all other areas are atomically flat with very low friction.

When there is no chemical interaction between the substrate and the counter-surface, the $0.34 \mathrm{~nm}$ thick graphene step edge exhibits a very small and symmetric topographic effect; such behavior is observed at the step edge covered by a single-layer of graphene (step-C in Fig. 1) [18]. The difference between these two cases-exposed versus buried step edges - reveals a very important clue about the super-lubricity. When there is no chemical interaction across the sliding interface, the additional resistive force during the ascending process is completely compensated by the assistive force during the descending process; so, small topographic corrugations of the surface without chemical interactions will not substantially deteriorate the super-lubricity. However, when two sliding surfaces have chemical interactions, then the resistive action during the ascending is significantly larger than the assistive action during the descending [17]. Thus, the super-lubricity will be destroyed even with the slightest topographic corrugations.

\section{Conclusions}

This paper reports how the friction behavior, which is measured for a single-layer thick graphene step edge using AFM, is affected by the scan condition as well as the tip bluntness. The AFM scan resolution must be high enough to capture the fine details of chemical and physical contributions of the step edge to the lateral signal, and averaging over multiple cycles is necessary to obtain such results with a good signal to noise ratio. The use of a blunt tip makes it easier to separate the chemical and physical components, allowing more quantitative analysis of each component, although it deteriorates the topographic image resolution.

\section{Acknowledgements}

This work was supported by the National Science
Foundation (Grant No. CMMI-1727571).

Open Access This article is licensed under a Creative Commons Attribution 4.0 International License, which permits use, sharing, adaptation, distribution and reproduction in any medium or format, as long as you give appropriate credit to the original author(s) and the source, provide a link to the Creative Commons license, and indicate if changes were made.

The images or other third party material in this article are included in the article's Creative Commons license, unless indicated otherwise in a credit line to the material. If material is not included in the article's Creative Commons license and your intended use is not permitted by statutory regulation or exceeds the permitted use, you will need to obtain permission directly from the copyright holder.

To view a copy of this license, visit http://creativecommons.org/licenses/by/4.0/.

\section{References}

[1] Berman D, Erdemir A, Sumant A V. Graphene: A new emerging lubricant. Mater Today 17(1): 31-42 (2014)

[2] Penkov O, Kim H J, Kim H J, Kim D E. Tribology of graphene: A review. Int J Precis Eng Manuf 15(3): 577-585 (2014)

[3] Kim K S, Lee H J, Lee C, Lee S K, Jang H, Ahn J H, Kim J H, Lee H J. Chemical vapor deposition-grown graphene: The thinnest solid lubricant. ACS Nano 5(6): 5107-5114 (2011)

[4] Berman D, Erdemir A, Sumant A V. Reduced wear and friction enabled by graphene layers on sliding steel surfaces in dry nitrogen. Carbon 59: 167-175 (2013)

[5] Berman D, Erdemir A, Sumant A V. Graphene as a protective coating and superior lubricant for electrical contacts. Appl Phys Lett 105(23): 231907 (2014)

[6] Wu P, Li X M, Zhang C H, Chen X C, Lin S Y, Sun H Y, Lin C T, Zhu H W, Luo J B. Self-assembled graphene film as low friction solid lubricant in macroscale contact. $A C S$ Appl Mater Interfaces 9(25): 21554-21562 (2017)

[7] Berman D, Deshmukh S A, Sankaranarayanan S K R S, Erdemir A, Sumant A V. Macroscale superlubricity enabled by graphene nanoscroll formation. Science 348(6239): 1118-1122 (2015)

[8] Liu S W, Wang H P, Xu Q, Ma T B, Yu G, Zhang C H, Geng D C, Yu Z W, Zhang S G, Wang W Z, et al. Robust microscale superlubricity under high contact pressure enabled by graphene-coated microsphere. Nat Commun 8: 14029 (2017) 
[9] Filleter T, McChesney J L, Bostwick A, Rotenberg E, Emtsev K V, Seyller T, Horn K, Bennewitz R. Friction and dissipation in epitaxial graphene films. Phys Rev Lett 102(8): 086102 (2009)

[10] Filleter T, Bennewitz R. Structural and frictional properties of graphene films on $\mathrm{SiC}(0001)$ studied by atomic force microscopy. Phys Rev B 81(15): 155412 (2010)

[11] Lee C, Li Q Y, Kalb W, Liu X Z, Berger H, Carpick R W, Hone J. Frictional characteristics of atomically thin sheets. Science 328(5974): 76-80 (2010)

[12] Lee H, Lee N, Seo Y, Eom J, Lee S. Comparison of frictional forces on graphene and graphite. Nanotechnology 20(32): 325701 (2009)

[13] Lee C, Wei X D, Li Q Y, Carpick R, Kysar J W, Hone J. Elastic and frictional properties of graphene. Phys Status Solidi B 246(11-12): 2562-2567 (2009)

[14] Li Q Y, Lee C, Carpick R W, Hone J. Substrate effect on thickness-dependent friction on graphene. Physi Status Solidi B 247(11-12): 2909-2914 (2010)

[15] Cho D H, Wang L, Kim J S, Lee G H, Kim E S, Lee S, Lee $\mathrm{S}$ Y, Hone J, Lee C. Effect of surface morphology on friction of graphene on various substrates. Nanoscale 5(7): 3063-3069 (2013)

[16] Paolicelli G, Tripathi M, Corradini V, Candini A, Valeri S. Nanoscale frictional behavior of graphene on $\mathrm{SiO}_{2}$ and $\mathrm{Ni}(111)$ substrates. Nanotechnology 26(5): 055703 (2015)

[17] Chen Z, Khajeh A, Martini A, Kim S H. Chemical and physical origins of friction on surfaces with atomic steps. Sci Adv 5(8): eaaw0513 (2019)

[18] Chen L, Chen Z, Tang X Y, Yan W M, Zhou Z R, Qian $\mathrm{L}$ M, Kim S H. Friction at single-layer graphene step edges due to chemical and topographic interactions. Carbon 154: 67-73 (2019)

[19] Lang H J, Peng Y T, Zeng X Z, Cao X A, Liu L, Zou K. Effect of relative humidity on the frictional properties of graphene at atomic-scale steps. Carbon 137: 519-526 (2018)

[20] Lang H J, Peng Y T, Zeng X Z. Effect of interlayer bonding strength and bending stiffness on 2-dimensional materials' frictional properties at atomic-scale steps. Appl Surf Sci 411: 261-270 (2017)

[21] Ye Z J, Martini A. Atomic friction at exposed and buried graphite step edges: Experiments and simulations. Appl Phys Lett 106(23): 231603 (2015)

[22] Lee H, Lee H B R, Kwon S, Salmeron M, Park J Y. Internal and external atomic steps in graphite exhibit dramatically different physical and chemical properties. ACS Nano 9(4): 3814-3819 (2015)

[23] Hunley D P, Flynn T J, Dodson T, Sundararajan A, Boland M J, Strachan D R. Friction, adhesion, and elasticity of graphene edges. Phys Rev B 87(3): 035417 (2013)

[24] Egberts P, Ye Z J, Liu X Z, Dong Y L, Martini A, Carpick R W. Environmental dependence of atomic-scale friction at graphite surface steps. Phys Rev B 88(3): 035409 (2013)

[25] Dong Y L, Liu X Z, Egberts P, Ye Z J, Carpick R W, Martini A. Correlation between probe shape and atomic friction peaks at graphite step edges. Tribol Lett 50(1): 49-57 (2013)

[26] Panigrahi S, Bhattacharya A, Bandyopadhyay D, Grabowski S J, Bhattacharyya D, Banerjee S. Wetting property of the edges of monoatomic step on graphite: Frictional-force microscopy and ab initio quantum chemical studies. $J$ Phys Chem C 115(30): 14819-14826 (2011)

[27] Hölscher H, Ebeling D, Schwarz U D. Friction at atomicscale surface steps: Experiment and theory. Phys Rev Lett 101(24): 246105 (2008)

[28] Müller T, Lohrmann M, Kässer T, Marti O, Mlynek J, Krausch G. Frictional force between a sharp asperity and a surface step. Phys Rev Lett 79(25): 5066-5069 (1997)

[29] Weilandt E, Menck A, Marti O. Friction studies at steps with friction force microscopy. Surf Interface Anal 23(6): 428-430 (1995)

[30] Ruan J A, Bhushan B. Frictional behavior of highly oriented pyrolytic graphite. J Appl Phys 76(12): 8117-8120 (1994)

[31] Schwoebel R L, Shipsey E J. Step motion on crystal surfaces. J Appl Phys 37(10): 3682-3686 (1966)

[32] Ye Z J, Otero-De-La-Roza A, Johnson E R, Martini A. Effect of tip shape on atomic-friction at graphite step edges. Appl Phys Lett 103(8): 081601 (2013)

[33] Barthel A J, Luo J W, Hwang K S, Lee J Y, Kim S H. Boundary lubrication effect of organic residue left on surface after evaporation of organic cleaning solvent. Wear 350-351: 21-26 (2016)

[34] Sader J E, Larson I, Mulvaney P, White L R. Method for the calibration of atomic force microscope cantilevers. Rev Sci Instrum 66(7): 3789-3798 (1995)

[35] Barnette A L, Asay D B, Janik M J, Kim S H. Adsorption isotherm and orientation of alcohols on hydrophilic $\mathrm{SiO}_{2}$ under ambient conditions. J Phys Chem C 113(24): 10632-10641 (2009)

[36] Yu J X, Kim S H, Yu B J, Qian L M, Zhou Z R. Role of tribochemistry in nanowear of single-crystalline silicon. ACS Appl Mater Interfaces 4(3): 1585-1593 (2012)

[37] Qi Y Z, Liu J, Dong Y L, Feng X Q, Li Q Y. Impacts of environments on nanoscale wear behavior of graphene: Edge passivation vs. Substrate pinning. Carbon 139: 59-66 (2018)

[38] Ogletree D F, Carpick R W, Salmeron M. Calibration of frictional forces in atomic force microscopy. Rev Sci Instrum 67(9): 3298-3306 (1996) 
[39] Varenberg M, Etsion I, Halperin G. An improved wedge calibration method for lateral force in atomic force microscopy. Rev Sci Instrum 74(7): 3362-3367 (2003)

[40] Noy A, Frisbie C D, Rozsnyai L F, Wrighton M S, Lieber C M. Chemical force microscopy: Exploiting chemically-modified tips to quantify adhesion, friction, and functional group distributions in molecular assemblies. J Am Chem Soc 117(30): 7943-7951 (1995)

[41] Dufrêne Y F, Ando T, Garcia R, Alsteens D, Martinez-Martin D, Engel A, Gerber C, Müller D J. Imaging modes of atomic force microscopy for application in molecular and cell biology.

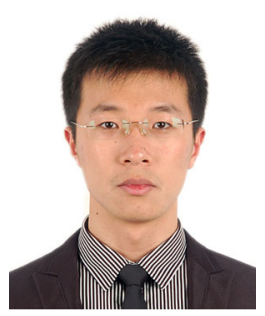

Zhe CHEN. Dr. Chen earned his B.S. degree in mechanical engineering from Northwestern Polytechnical University and Ph.D. degree in mechanical engineering from

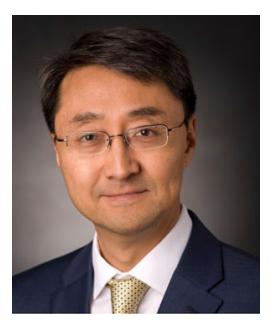

Seong H. KIM. Prof. Kim joined the Faculty of Chemical Engineering in 2001 after completing a Ph.D. study in chemistry from Northwestern University and a postdoctoral research at University of California, Berkeley, USA. He earned
Nat Nanotechnol 12(4): 295-307 (2017)

[42] Levita G, Restuccia P, Righi M C. Graphene and $\mathrm{MoS}_{2}$ interacting with water: A comparison by ab initio calculations. Carbon 107: 878-884 (2016)

[43] Mo Y, Turner K T, Szlufarska I. Friction laws at the nanoscale. Nature 457: 1116-1119 (2009)

[44] Chen Z, Vazirisereshk M R, Khajeh A, Martini A, Kim S H. Effect of atomic corrugation on adhesion and friction: A model study with graphene step edges. J Phys Chem Lett 10(21): 6455-6461 (2019)

Tsinghua University in China. He is now a postdoctoral researcher. His research interests are interfacial sliding properties and applications of two-dimensional layered materials as solid lubricants and lubricant additives.

his B.S. and M.S. degrees in chemistry from Yonsei University, Korea. The main theme of his research lies in surface science and nano-engineering, especially applying surface science fundamentals to important interfacial problems involved in tribology and lubrication, nanomanufacturing, and surface engineering. 